\title{
NeuroImage
}

\section{Constrained linear basis sets for HRF modelling using Variational Bayes}

\author{
Mark W. Woolrich, ${ }^{\mathrm{a}, \mathrm{b}, *}$ Timothy E.J. Behrens, ${ }^{\mathrm{a}, \mathrm{b}}$ and Stephen M. Smith ${ }^{\mathrm{a}}$ \\ ${ }^{a}$ Oxford Centre for Functional Magnetic Resonance Imaging of the Brain (FMRIB), University of Oxford, John Radcliffe Hospital, Headington, \\ Oxford OX3 9DU, UK \\ ${ }^{\mathrm{b}}$ Department of Engineering Science, University of Oxford, Oxford, UK
}

Received 4 September 2003; revised 6 December 2003; accepted 9 December 2003

\begin{abstract}
FMRI modelling requires flexible haemodynamic response function (HRF) modelling, with the HRF being allowed to vary spatially and between subjects. To achieve this flexibility, voxelwise parameterised HRFs have been proposed; however, inference on such models is very slow. An alternative approach is to use basis functions allowing inference to proceed in the more manageable General Linear Model (GLM) framework. However, a large amount of the subspace spanned by the basis functions produces nonsensical HRF shapes. In this work we propose a technique for choosing a basis set, and then the means to constrain the subspace spanned by the basis set to only include sensible HRF shapes. Penny et al. [NeuroImage (2003)] showed how Variational Bayes can be used to infer on the GLM for FMRI. Here we extend the work of Penny et al. to give inference on the GLM with constrained HRF basis functions and with spatial Markov Random Fields on the autoregressive noise parameters. Constraining the subspace spanned by the basis set allows for far superior separation of activating voxels from nonactivating voxels in FMRI data. We use spatial mixture modelling to produce final probabilities of activation and demonstrate increased sensitivity on an FMRI dataset.

(C) 2004 Elsevier Inc. All rights reserved.
\end{abstract}

Keywords: Variational Bayes; GLM; FMRI; HRF; Basis sets; Markov random field

\section{Introduction}

An important issue for detecting areas of brain activity in FMRI is the forward modelling of the temporal BOLD response. This forward model predicts what the BOLD response would be if we knew the underlying neural activity.

To allow modelling of BOLD responses to general stimulation types, Friston et al. (1994) introduced the use of convolution models, which assume a linear time invariant system. Boynton et

* Corresponding author. Oxford Centre for Functional Magnetic Resonance Imaging of the Brain (FMRIB), University of Oxford, John Radcliffe Hospital, Headington, Oxford OX3 9DU, UK. Fax: +44-1865222717.

E-mail address: woolrich@fmrib.ox.ac.uk (M.W. Woolrich).

Available online on ScienceDirect (www.sciencedirect.com.) al. (1996), Cohen (1997), Dale and Buckner (1997), and provide some evidence that the BOLD response possesses linear characteristics with respect to the stimulation. However, nonlinearities are predominant when there are short separations (less than approximately $3 \mathrm{~s}$ ) between stimuli (Friston et al., 1998b). An additional assumption is that the stimulus represents the underlying neural activity. The stimulus (or neural activity) is then convolved with an assumed or modelled impulse response function, known as haemodynamic response function (HRF), to give the assumed BOLD response (Friston et al., 1994).

Genovese (in press) and Gössl et al. (2001) have previously used a Bayesian framework to model the voxelwise BOLD response to a sustained period of stimulation. The advantage of a Bayesian approach is most obvious in the use of prior experience to justify the prior distributions used for these haemodynamic response parameters. However, the work of Genovese (in press) and Gössl et al. (2001) was restricted to just modelling the response to an epoch of fixed size. Woolrich et al. (in press) generalised this to general stimulations via parametric modelling of the HRF assuming a linear time-invariant system. However, the problem with parametric modelling of the HRF is that the model is difficult to infer upon without slow techniques such as Markov Chain Monte Carlo (MCMC).

Friston et al. (1995) and Josephs et al. (1997) consider voxelwise linear time-invariant system HRF models within the framework of the General Linear Model (GLM). Flexibility to model the HRF is introduced via basis sets. However, a large amount of the subspace spanned by the basis functions produces nonsensical HRF shapes [see Fig. 4(a)]. This is because the conventional GLM will indiscriminately allow all possible linear combinations of the basis set.

In this work we propose a technique for using soft constraints to weight the subspace spanned by the basis set to only include sensible HRF shapes within a linear time-invariant system. The choice of the basis set can be driven by a standard parametric HRF, or a physiologically informed model such as the Balloon Model (Buxton et al., 1998). Using the GLM in a Bayesian framework, we can then use priors on the basis function regression parameters to constrain the linear combinations of HRFs to sensible HRF shapes. Penny et al. (2003) showed how Variational Bayes can be used to infer on the GLM for FMRI. Here we extend this work to 
give inference on the GLM with the constrained HRF basis functions. We also extend the work of Penny et al. (2003) to spatially regularise the autoregressive noise parameters using a Markov Random Field (MRF). We demonstrate that the constrained basis function approach allows for far superior sensitivity, when compared with traditional unconstrained basis function approaches.

\section{Overview}

We start in the "Model" section by describing how the GLM in a fully Bayesian framework allows us to introduce soft constraints on linear combinations within the GLM. We then discuss how we choose a sensible basis set, and determine the required basis set constraints. In the "Inference" section we describe how we perform approximate Variational Bayesian inference on the model. In the "Artificial null data" section we use the model on artificial null data to demonstrate the effect of the HRF constraints. Then in the "Spatial mixture modelling" section we describe how we can use spatial mixture modelling to produce probabilities of activation, which takes advantage of the extra sensitivity produced from the HRF constraints. Finally, in the "FMRI data" section we demonstrate this increased sensitivity on an audiovisual dataset.

\section{Model}

Here we describe the GLM in the fully Bayesian framework. It will be via the priors on the regression parameters that we propose to constrain the possible linear combinations that are allowed.

Consider that the preprocessed FMRI data at voxel $i$ and at scan $t$ is $y_{i t}(i=1 \ldots N, t=1 \ldots T)$, the $t$ th $1 \times K$ row of the design matrix, $x$, is $x_{t}$, and $\beta_{i}$ is a $K \times 1$ vector of parameter estimates. The preprocessed FMRI data, $y$, is taken to have been motion corrected and high-pass filtered. The standard general linear model (GLM) is then:

$y_{i t}=x_{t} \beta_{i}+\eta_{i t}$

We model the error, $\eta_{i t}$, as a voxelwise temporal autoregressive process of order $P(\operatorname{AR}(P))$. We can represent this as:

$\eta_{i t}=\sum_{p=1}^{P} a_{p i} \eta_{i(t-p)}+e_{i t}$

$e_{i t} \sim N\left(0, \phi_{e_{i}}^{-1}\right)$

where $a_{p i}$ is the $p$ th AR coefficient $(p=1, \ldots, P)$.

Bayesian framework

In this work we use a Bayesian framework. Eqs. (1) and (2) form our likelihood. The distribution we are interested in is the full posterior distribution over the model parameters, and depends upon this likelihood and the priors over the unknown parameters in our model via Bayes rule:

$p\left(\beta, a, \phi_{e} \mid y\right) \propto \prod_{i} p\left(y_{i} \mid \beta_{i}, a_{i}, \phi_{e_{i}}\right) p\left(\beta, a, \phi_{e}\right)$
We now need to consider the specification of priors over the parameters in our model. A priori we assume independence between the parameters:

$p\left(\beta, a, \phi_{e}\right)=p(\beta) p(a) p\left(\phi_{e}\right)$

As we shall see, using independent priors allows us to use conjugate priors, which in turn makes the model tractable when using Variational Bayes. However, assuming that we have independence between priors for different parameters does not mean that the parameters will be independent in the posterior. Any dependence between parameters inferred from the data and the likelihood will still be reflected in the joint posterior. For the precision we assume a voxelwise noninformative Gamma prior:

$p\left(\phi_{e}\right)=\prod_{i} p\left(\phi_{e_{i}}\right)$

$\phi_{e_{i}} \sim G a\left(b_{e_{0}}, c_{e_{0}}\right)$

Autoregressive parameters spatial prior

In Penny et al. (2003) nonspatial noninformative priors were used on the autoregressive parameters. Previous work has shown that neighbouring voxels have similar temporal autocorrelation (Woolrich et al., 2001; Worsley et al., 2002). Therefore, we want to model the assumption that a priori we expect neighbouring voxels to have similar temporal autocorrelation. To do this we use a Gaussian conditionally specified autoregressive (CAR) or continuous Markov Random Field (MRF) prior (Cressie, 1993) on each of the $P$ autoregressive parameter maps; that is, $p(a)=\prod_{p=1}^{P} p\left(a_{p}\right)$ with:

$p\left(a_{p} \mid \phi_{a_{p}}\right) \sim \operatorname{MVN}\left(0, \phi_{a_{p}}^{-1} D^{-1}\right)$

where MVN denotes a multivariate Normal distribution, $D$ is an $N$ $\times N$ matrix whose $(i, j)$ th element is $d_{i j}$, and $\phi_{a_{p}}$ is the MRF control parameter that controls the amount of spatial regularisation. We set $d_{i i}=1, d_{i j}=-1 / N_{i j}$ if $i$ and $j$ are spatial neighbours and $d_{i j}=0$ otherwise (where $N_{i j}$ is the geometric mean of the number of neighbours for voxels $i$ and $j$ ). We also require a hyperprior on $\phi_{a_{p}}$, for which we use a standard noninformative conjugate Gamma prior:

$\phi_{a_{p}} \sim G a\left(b_{a_{0}}, c_{a_{0}}\right)$

Before we specify the prior for the regression parameters $\beta$, we consider how we can use basis functions within this framework.

Basis functions

In this work we model the different HRF shapes for different underlying conditions at different voxels using basis functions and assuming a linear time-invariant system (Josephs et al., 1997). If we have $e=1 \ldots N_{e}$ underlying conditions, and for each condition we have $b=1 \ldots N_{b}$ basis functions to model the HRF for that condition for voxel $i$, we can rewrite Eq. (1) as:

$y_{i t}=\sum_{e=1}^{N_{e}} \sum_{b=1}^{N_{b}}\left\{x_{e b t} \beta_{i e b}\right\}+\eta_{i t}$ 
where $\beta_{i e b}$ is the regression parameter for the $b$ th basis function of the eth underlying condition at voxel $i$ and:

$x_{e b \tau}=g_{b \tau} \otimes s_{e \tau}$

where $\otimes$ represents convolution, $g_{b \tau}$ is the $b$ th basis function and $s_{e \tau}$ is the eth stimulus function. Note that $\tau$ is an index at higher temporal resolution than $t$, to capture all of the HRF shape (i.e., for a resolution of $1 / \rho$ of a TR, $\tau=1 \ldots \rho T$ ). We obtain $x_{e b t}$ from $x_{e b \tau}$ by sampling every $\rho$ th $x_{e b \tau}$.

\section{Constraining basis function linear combinations}

Here we are going to describe how we constrain the basis function linear combinations. To do this we need to reparameterise the regression parameters, $\beta_{i e b}$, into parameters that describe the shape of the HRF, and parameters that scale these HRF shape parameters, to give the actual fit in the GLM.

Firstly, we specify $\beta_{i e}$ as the $N_{b} \times 1$ vector of the regression parameters for the $N_{b}$ basis functions for the eth underlying condition at voxel $i$. Then, we reparameterise $\beta_{i e}$ as being:

$$
\beta_{i e}=\bar{D}_{i e} \frac{D_{i e}}{\sqrt{\left(\sum_{b} D_{i e b}^{2} / N_{b}\right)}}
$$

where $D_{i e}$ is an $N_{b} \times 1$ vector of parameters describing the HRF for underlying condition $e$, and $\bar{D}_{i e}$ is the scalar value representing the scaling of that HRF. We want the scalar $\bar{D}_{i e}$ to contain all of our size information. However, left unchecked, there is an arbitrary scale factor on vector $D_{i e}$. We have removed this arbitrary scale factor by normalising the vector using its root mean square. Hence, we now have a normalised vector, $D_{i e} / \sqrt{\sum_{b} D_{i e b}^{2} / N_{b}}$, representing the shape of the HRF, and a scalar, $\bar{D}_{i e}$, representing the size of the HRF.

For the scaling parameters, we assume a noninformative prior:

$p(\bar{D})=\prod_{i e} p\left(\bar{D}_{i e}\right)$

$\bar{D}_{i e} \sim N\left(0, \phi_{\bar{D}_{0}}^{-1}\right)$

where the precision, $\phi_{\bar{D}_{0}}$, is fixed to be very small $(1 \mathrm{e}-6)$ for all voxels. It is via the prior on $D_{i e}$ that we can constrain the possible linear combinations of basis functions to represent the HRF for an underlying condition. We specify the prior on $D_{i e}$ as:

$p(D)=\prod_{i e} p\left(D_{i e}\right)$

$D_{i e} \sim \operatorname{MVN}\left(m, C^{-1}\right)$

where $m$ and $C$ will contain the information constraining the possible linear combinations of the basis functions (see "Determining basis set constraints" for how we set $m$ and $C$ ).

\section{Choosing a basis set}

Basis sets used previously range from a single canonical HRF plus its temporal derivative to a set of Gamma functions (Friston et al., 1998a). These basis functions are then separately convolved with the known stimulus to give the same number of regressors as there are basis functions for use in the linear model.

Hossein-Zadeh and Ardekani (2002) and Friman et al. (2003) have previously shown how we can generate a basis set using singular value decomposition (SVD). This produces a basis set from samples of the HRF or regressors resulting from a parametric forward model of the haemodynamics. This is the approach we take in this paper.

In this paper we base the basis set on a parameterised model of the HRF. The HRF is parameterised by four half-cosines, requiring six parameters, as illustrated in Fig. 1.

To complete the HRF parameterised model, we need to specify probabilities for the parameter values in the HRF model from which we generate physiologically plausible HRF shapes. With this information, we can then draw HRF samples. Fig. 2 shows 20 HRF samples drawn from the half-cosine parameterisation using the HRF parameter value probabilities:

$h_{1} \sim \operatorname{Uniform}(0 s, 2 s)$

$h_{2} \sim \operatorname{Uniform}(2 s, 6 s)$

$h_{3} \sim \operatorname{Uniform}(2 s, 6 s)$

$h_{4} \sim$ Uniform $(2 s, 8 s)$

$f_{1}=0$

$f_{2} \sim \operatorname{Uniform}(0,0.5)$

We strongly emphasise that this is one of many possible choices of obtaining HRF samples. An alternative, attractive approach would be to use a physiologically based model, such as the Balloon model (Friston et al., 2000), with physiologically meaningful parameters that can be given sensible ranges.

Using a probabilistic model of choice, one can obtain a set of samples of the HRF, which represents the space of HRFs expected. The $N_{H}$ HRF samples, each of length $N_{T}=\rho T$, can be placed into a $N_{T} \times N_{H}$ matrix, $W$. To obtain a basis set that spans this space of HRFs, we perform a Singular Value Decomposition (SVD) on this matrix. This gives us $N_{H}$ eigen-HRFs (of length $N_{T}$ ) and $N_{H}$ corresponding eigenvalues (describing the power each corresponding eigen-HRF explains).

Fig. 3 shows the four eigen-HRFs (and their corresponding eigenvalues) with the largest eigenvalues obtained from an SVD on $N_{H}=1000 \mathrm{HRF}$ samples of length $N_{T}=512$ (resolution of $0: 5 \mathrm{~s}$ ) from the half-cosine parameterisation. It is worth noting that the first three eigen-HRFs/basis functions look remarkably like the commonly used canonical, delay (temporal) derivative and width (dispersion) derivative of a Gamma/Gaussian parameterised HRF (in that order).

To form our basis set, we need to decide how many of the largest eigen-HRFs we want to include. For the rest of this paper we use the $N_{b}=3$ largest eigen-HRFs as our basis set.

\section{Determining basis set constraints}

In the previous section we determined a basis set to use. In this section we describe how we can apply constraints on the linear combinations of the basis set. In a previous work, Friman et al. 


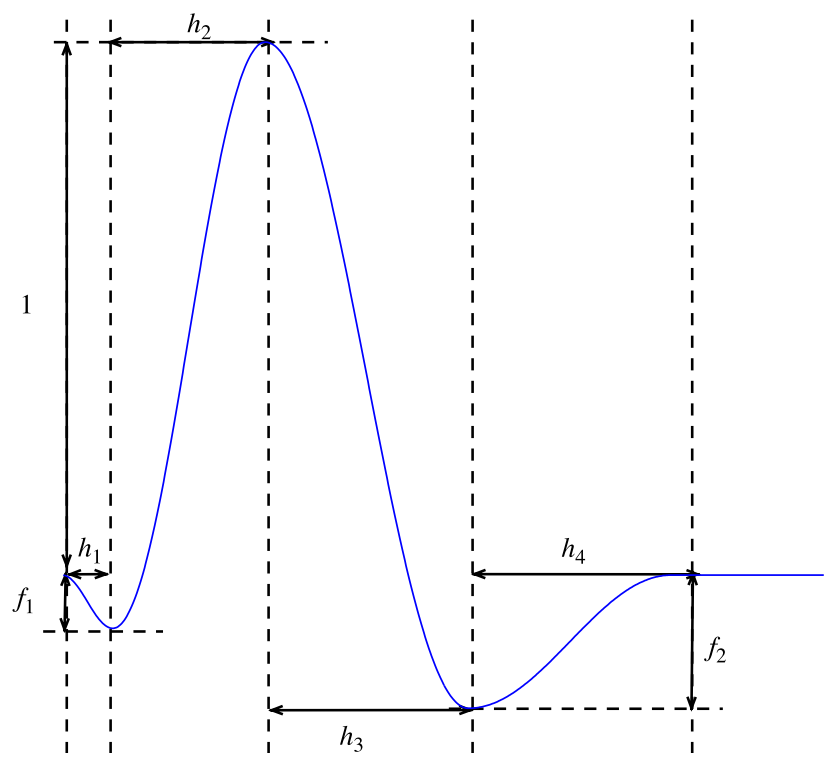

Fig. 1. Parameterisation of the HRF into four half-period cosines. There are six parameters.

(2003) also looked to constrain the possible linear combinations of the basis set, but within the canonical correlation analysis framework. However, they only looked to constrain the linear combination coefficients to be positive. In this work we look to apply a more complete constraint by fitting a multivariate Normal distribution to describe the desired constrained space probabilistically within the GLM framework.

To date, basis sets are used by convolving the constituent basis functions with the known stimulus to give the same number of regressors as there are basis functions stimuli. The resulting regressors are then used in the linear model. This approach corresponds to the model we have described earlier but with $m=0$ and $C=I$ in Eq. (13). Fig. 4(a) shows example HRF shapes randomly drawn from this basis set when the linear combinations are unconstrained (i.e., with $m=0$ and $C=I$ ). If we compare these HRFs with those in Fig. 2, it is clear that unconstrained linear combinations of our basis set allows for nonsensical HRF shapes.

To provide constraints on the possible linear combinations, we regress the HRF samples we used to obtain our basis set back onto the basis set:

$W=G R+e$

where $W$ is the $N_{T} \times N_{H}$ matrix containing our $N_{H}$ HRF samples, $G$ is the $N_{T} \times N_{b}$ matrix of our $N_{b}$ basis functions and $e \sim N\left(0, \sigma^{2} I\right)$. We can perform standard Ordinary Least Squares (OLS) to obtain an estimate of the $N_{b} \times N_{H}$ matrix, $R$ :

$\hat{R}=\left(G^{T} G\right)^{-1} G^{T} W$

We then fit an $N_{b}$-dimensional multivariate Normal distribution, $\operatorname{MVN}(\tilde{m}, \tilde{C})$, to the matrix $\hat{R}$. The parameters of this multivariate Normal distribution are used to set the parameters in Eq. (13) (i.e., $m=\tilde{m}$ and $C=\tilde{C}$ ).

Using the HRF parameter value probabilities in Eq. (14) for our half-cosine HRF parameterisation, 1000 resulting HRF samples, and the resulting top three eigen-HRFs, we obtain the multivariate Normal distribution parameters:

$$
\begin{aligned}
& \tilde{m}=[0.86,0.09,0.01]^{T} \\
& \tilde{C}=\left[\begin{array}{ccc}
0.018 & 0.028 & -0.015 \\
0.028 & 0.185 & -0.009 \\
-0.015 & -0.009 & 0.030
\end{array}\right]
\end{aligned}
$$

Fig. 4(b) shows example HRF shapes obtained randomly drawn from this basis set when the linear combinations are constrained with these $m=\tilde{m}$ and $C=\tilde{C}$. We can see that compared with Fig. 4(a), this is a much more faithful representation of the sensible HRF shapes in Fig. 2.

It is important to point out that constraining the linear combinations by using a multivariate Normal distribution is an approximation. This is because a multivariate Normal will not capture all of the detail of the distribution in the $N_{b}$ parameter space. As Fig. 4(b) shows, while we are producing much more sensible shapes than the unconstrained case, there are still some undesirable HRF shapes. The alternative is a fully parametric HRF approach (Woolrich et al., in press), which is very slow to infer upon, or a different distribution to a multivariate Normal that can capture the required detail in the $N_{b}$ parameter space. However, we choose to use a multivariate Normal as it will make inference much easier to handle within the Variational Bayesian framework, and it does capture most of the required structure.

\section{Inference}

The distribution we are interested in inferring upon is the posterior distribution $p(\theta \mid y)$ (Eq. (3)), where $\theta$ is the set of parameters $\left\{\beta, a, \phi_{e}\right\}$. It is not possible to solve for this distribution analytically. Hence we use the framework introduced to FMRI by



Fig. 2. Twenty HRF samples drawn from the half-cosine parameterisation. 

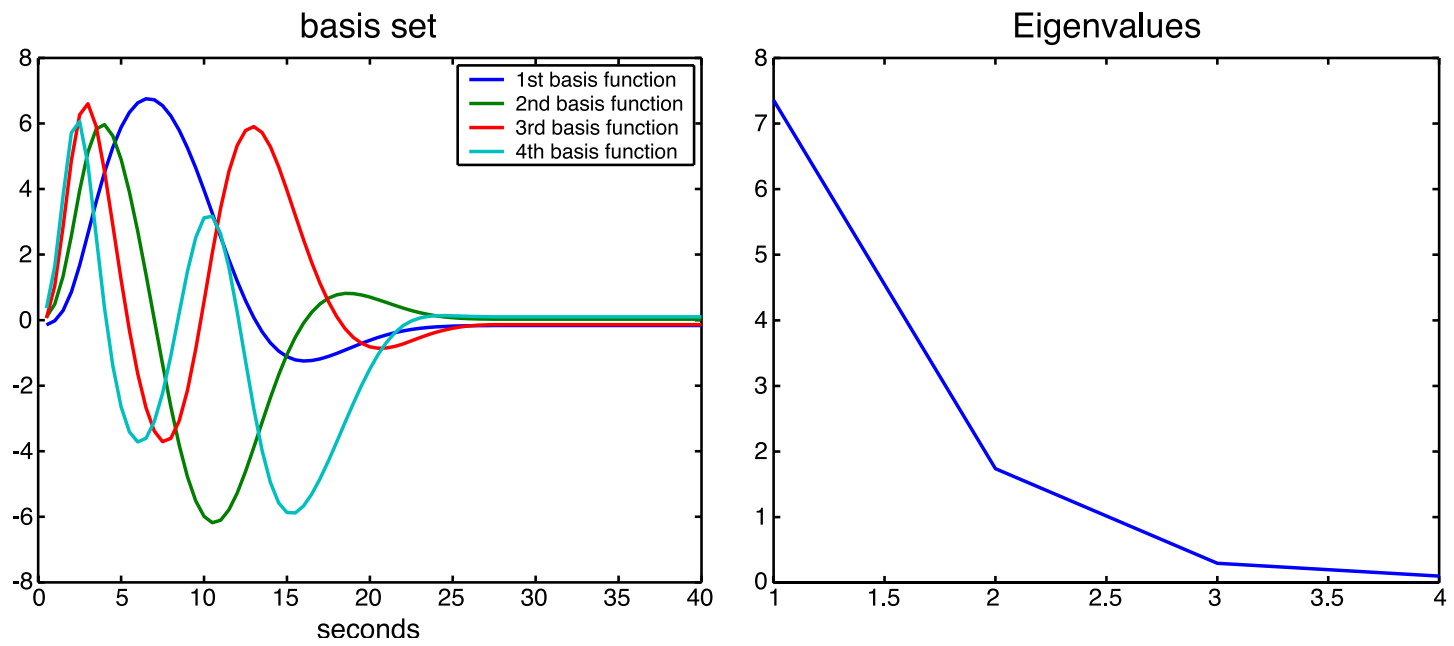

Fig. 3. Four eigen-HRFs (and their corresponding eigenvalues) with the largest eigenvalues from $N_{H}=1000 \mathrm{HRF}$ samples of length $N_{T}=512$ (resolution of $0.5 \mathrm{~s}$ ) from the half-cosine parameterisation.

(a)

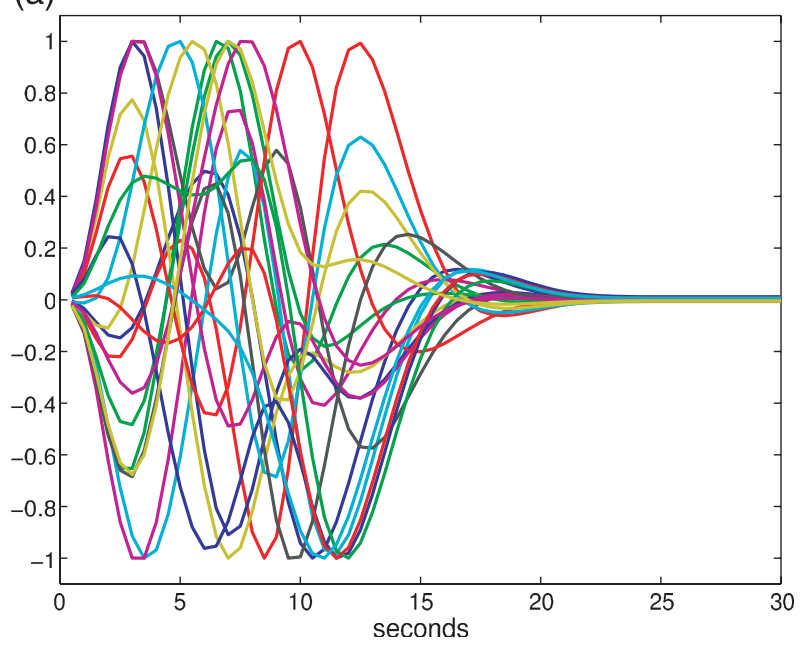

(b)

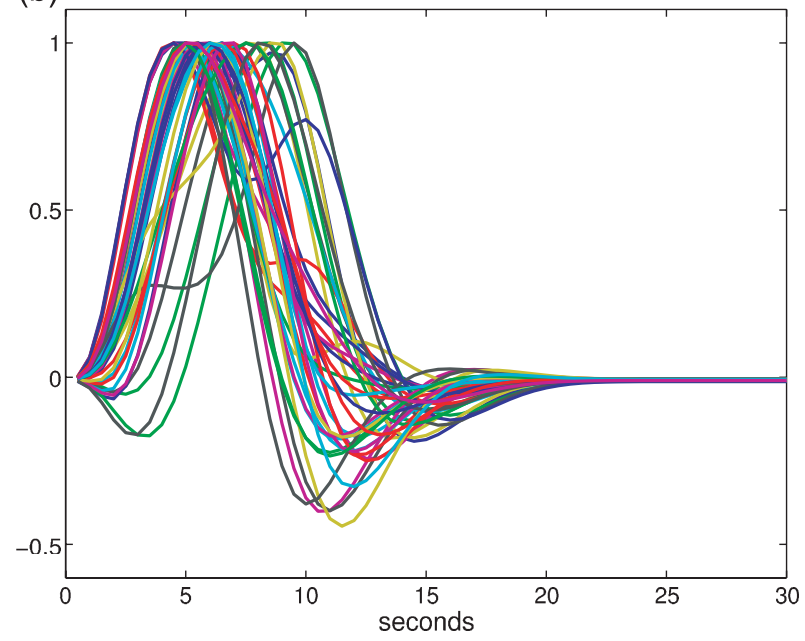

Fig. 4. Samples from the basis set: (a) unconstrained with $m=0$ and $C=I$; (b) constrained with $m=\tilde{m}$ and $C=\tilde{C}$. (a)

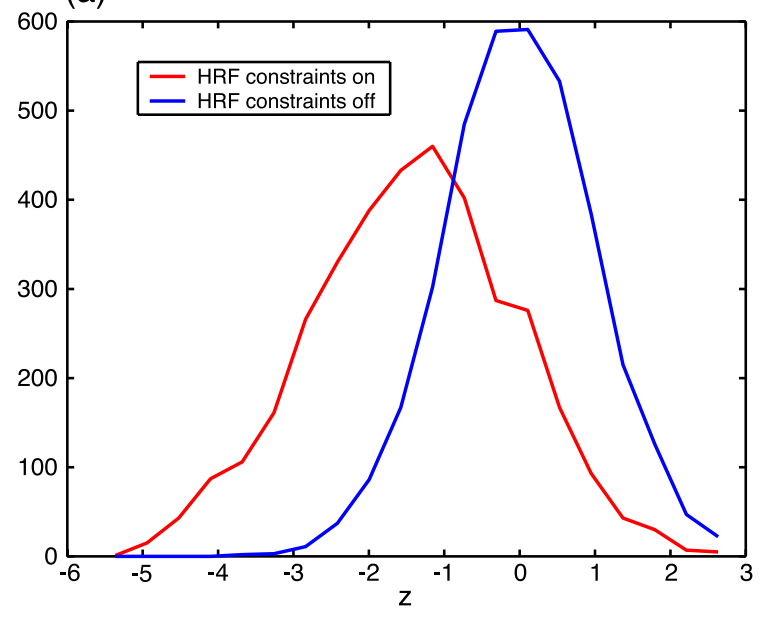

(b)

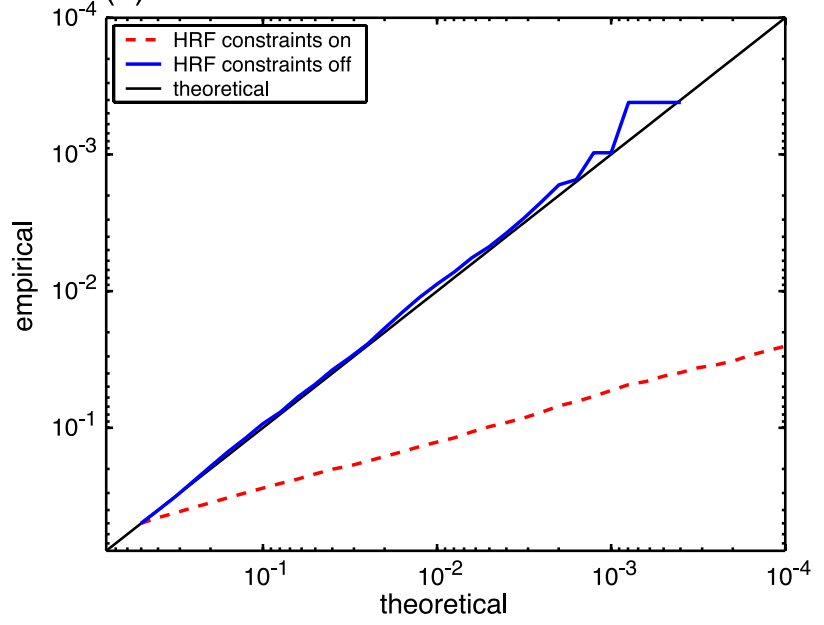

Fig. 5. (a) Histogram of pseudo-z-statistics obtained for the two different models with and without HRF constraints. (b) Log probability-log probability plots. These show plots of (nominal/theoretical) frequentist FPR against that obtained empirically. The HRF constraints reduce the power in those voxels where the linear combinations of the basis functions do not give sensible HRF shapes; this produces a shift in the null distribution histogram. 


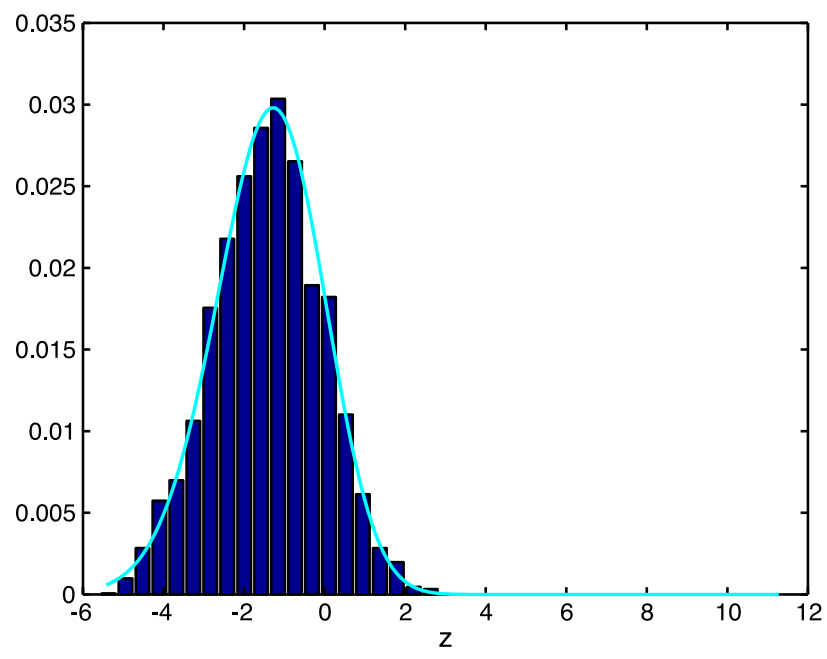

Fig. 6. Null distribution of pseudo-z-statistics resulting from the constrained HRF analysis on the artificial null data. This is the same histogram as that shown in red in Fig. 5(a). Also shown is the fit to this histogram of a flipped and shifted Gamma distribution.

Penny et al. (2003) of Variational Bayes. For a general introduction to Variational Methods, see Jordan (1999). Using this approach we can approximate a posterior distribution $p(\theta \mid y)$ with $q(\theta \mid y)$ by minimising the KL-divergence, or equivalently by maximising the variational free energy, $F$, between them:

$F=\int q(\theta \mid y) \log \frac{p(y, \theta)}{q(\theta \mid y)} \mathrm{d} \theta$

To maximise this function, we need to ensure that the resulting integrals are tractable. A standard way to help achieve this is to use conjugate priors and to factorise the approximate posterior.

In the modelling section, we parameterised the model in terms of parameters $a, \phi_{e}, D$ and $\bar{D}$, and wherever possible specified

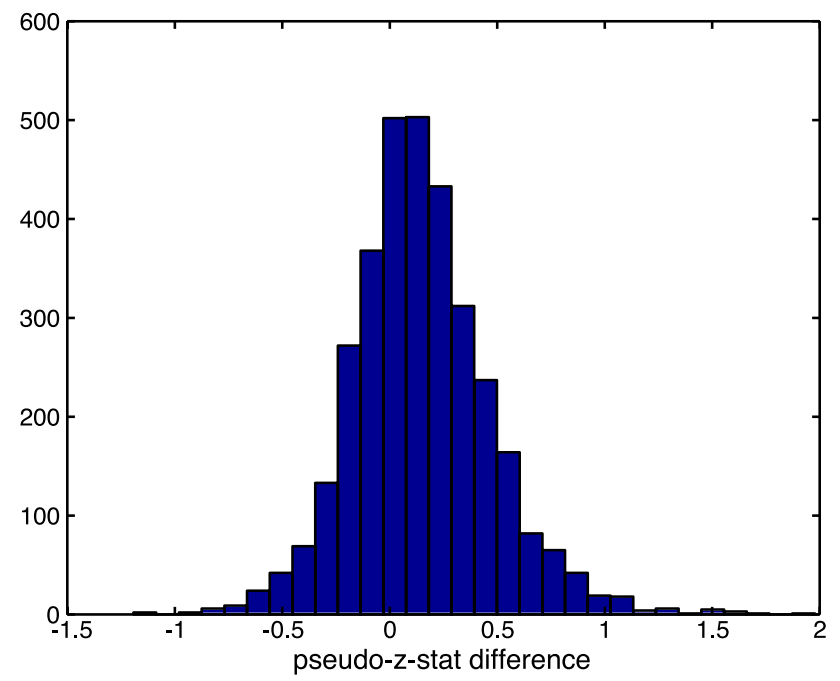

Fig. 8. Histogram of the voxelwise difference in pseudo-z-statistics between the model with nonspatial noninformative AR priors and the model with adaptive spatial MRF AR priors for the single-event pain dataset.

conjugate priors on them. However, using these parameters and factorising the posterior into

$$
\begin{aligned}
& q\left(D, \bar{D}, a, \phi_{a}, \phi_{e} \mid y\right) \\
& \quad=\prod_{p}\left\{q\left(a_{p} \mid y\right) p\left(\phi_{a_{p}} \mid y\right)\right\} \prod_{i}\left\{q\left(D_{i} \mid y\right) q\left(\bar{D}_{i} \mid y\right) q\left(\phi_{e_{i}} \mid y\right)\right\}
\end{aligned}
$$

is not tractable to Variational Bayes as we cannot derive the update equations for $q\left(D_{i e} \mid y\right)$ and $q\left(\bar{D}_{i e} \mid y\right)$. To overcome this problem, instead of using the two parameters $D_{i e}$ and $\bar{D}_{i e}$, we reparameterise to use $\beta_{i e}$ and $\bar{\beta}_{i e}$ by rewriting Eq. (11) as:

$\beta_{i e}=\bar{\beta}_{i e} D_{i e}$
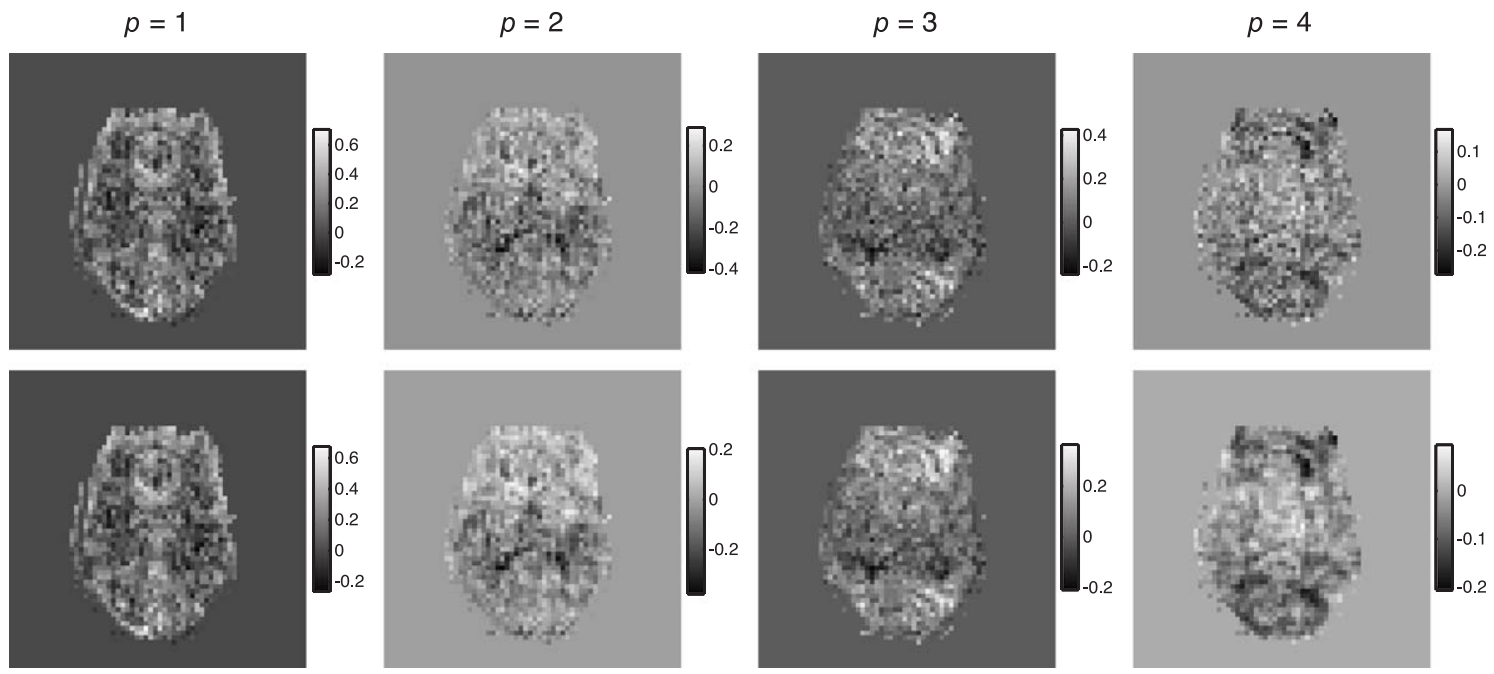

Fig. 7. Posterior means of parameters from an autoregressive model of order 4 from the single-event pain dataset. From left to right, we have increasing $p$ from 1 to 4 . We show this for two different priors on the AR parameters: (top) noninformative nonspatial, and (bottom) spatial MRF. 
(a) Visual boxcar

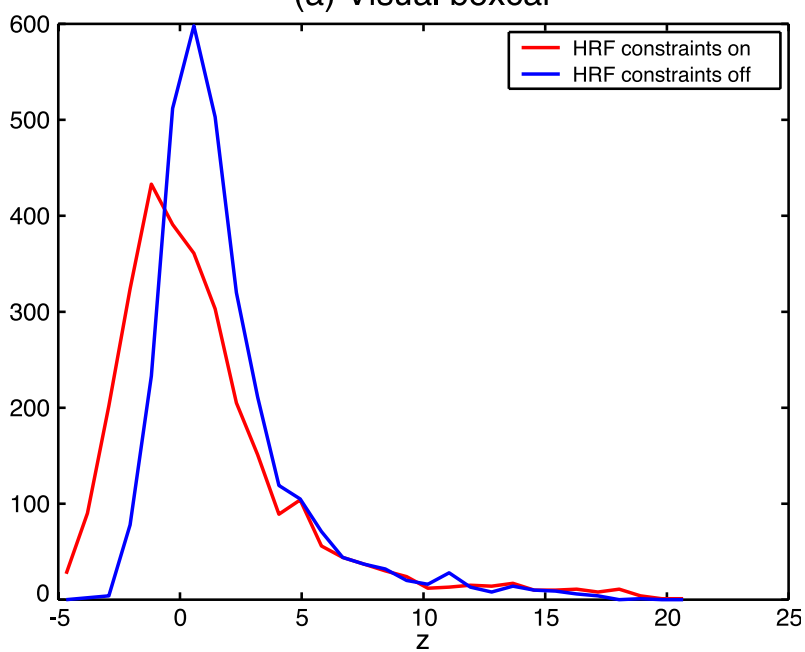

(b) Pain single-event

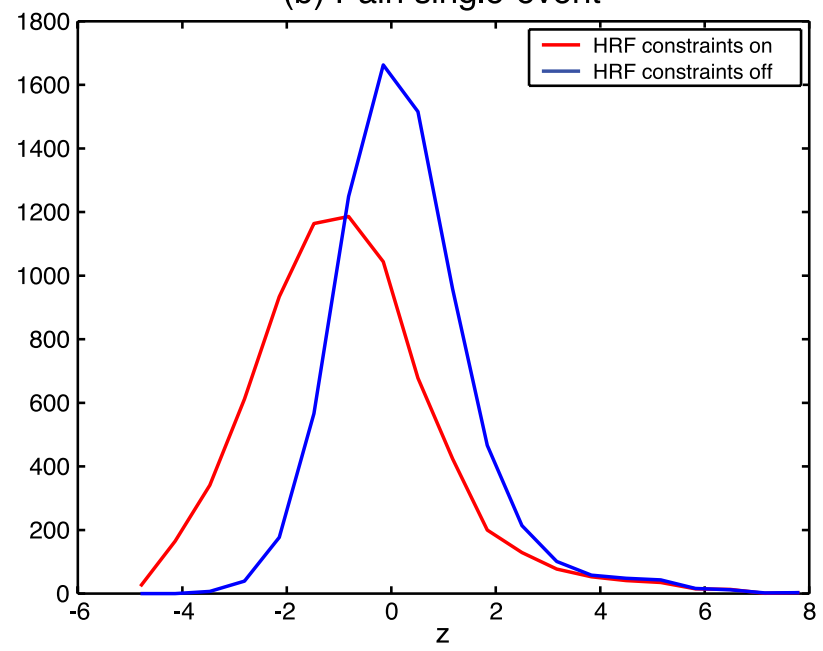

Fig. 9. Histogram of pseudo-z-statistics obtained for two different models (with and without HRF constraints) on (a) the visual boxcar dataset and (b) the pain single-event dataset. where

$$
\bar{\beta}_{i e}=\frac{\bar{D}_{i e}}{\sqrt{\left(\sum_{b} D_{i e b}^{2} / N_{b}\right)}} .
$$

Recall from Eq. (11) that $\sqrt{\sum_{b} D_{i e b}^{2} / N_{b}}$ is the normalisation on the HRF shape vector, $D_{i e}$, and the scalar, $\bar{D}_{i e}$, represents the size of the HRF. We now have the following prior on $\beta_{i e}$ :

$\beta_{i e} \mid \bar{\beta}_{i e} \sim \operatorname{MVN}\left(\bar{\beta}_{i e} m, \bar{\beta}_{i e}^{2} C^{-1}\right)$

and a noninformative prior on $\beta_{i e}$ :

$\bar{\beta}_{i e} \sim N\left(0, \phi_{\bar{\beta}_{0}}^{-1}\right)$

where the precision, $\phi_{\beta_{0}}^{-}$, is fixed to be very small $(1 \mathrm{e}-6)$ for all voxels. We now assume the following factorised form for the approximate posterior:

$$
\begin{aligned}
& q\left(\beta, \bar{\beta}, a, \phi_{e} \mid y\right) \\
& \quad=\prod_{p}\left\{q\left(a_{p} \mid y\right) p\left(\phi_{a_{p}} \mid y\right)\right\} \prod_{i}\left\{q\left(\beta_{i}, \bar{\beta}_{i} \mid y\right) q\left(\phi_{e_{i}} \mid y\right)\right\}
\end{aligned}
$$

where

$$
\begin{aligned}
& q\left(B_{i} \mid y\right)=\operatorname{MVN}\left(\mu_{B_{i}}, \Lambda_{B_{i}}\right) \\
& q\left(a_{p} \mid y\right)=N\left(\mu_{a_{p}}, \Lambda_{a_{p}}\right) \\
& q\left(\phi_{a_{p}} \mid y\right)=G a\left(b_{a_{p}}, c_{a_{p}}\right) \\
& q\left(\phi_{e_{i}} \mid y\right)=G a\left(b_{e_{i}}, c_{e_{i}}\right)
\end{aligned}
$$

where $B_{i}=\left(\beta_{i}, \bar{\beta}_{i}\right)$, where $\beta_{i}$ is the $\left(N_{e} N_{b}\right) \times 1$ vector $\left[\beta_{i 1} \ldots \beta_{i e} \ldots\right.$ $\left.\beta_{i N}\right]^{T}$ and $\bar{\beta}_{i}$ is the $N e \times 1$ vector $\left[\bar{\beta}_{i 1}, \ldots \bar{\beta}_{i e} \ldots \bar{\beta}_{i N}\right]^{T}$. Note that we do not fully factorise. We would not expect $\beta_{i}$ and $\bar{\beta}_{i}$ to be
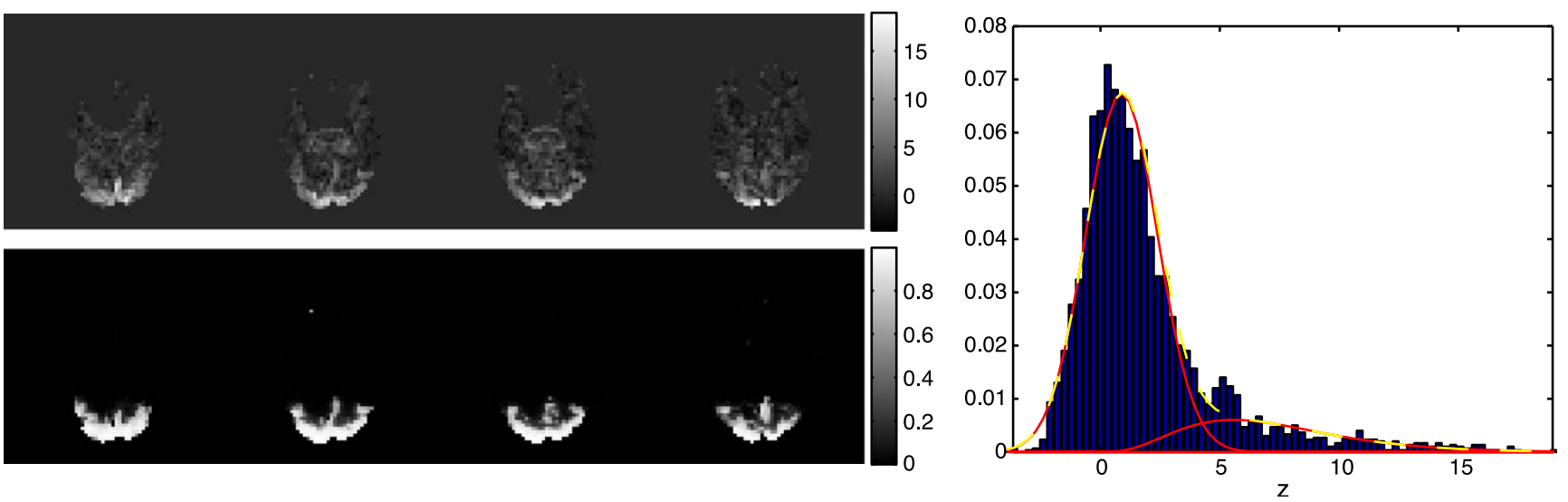

Fig. 10. Visual condition. Results of applying the spatial mixture modelling on the unconstrained HRF model. (top) Pseudo-z-statistic spatial maps. (bottom) Probability of being in the activation class. (right) Mixture model fit to histogram of pseudo-z-statistics. 

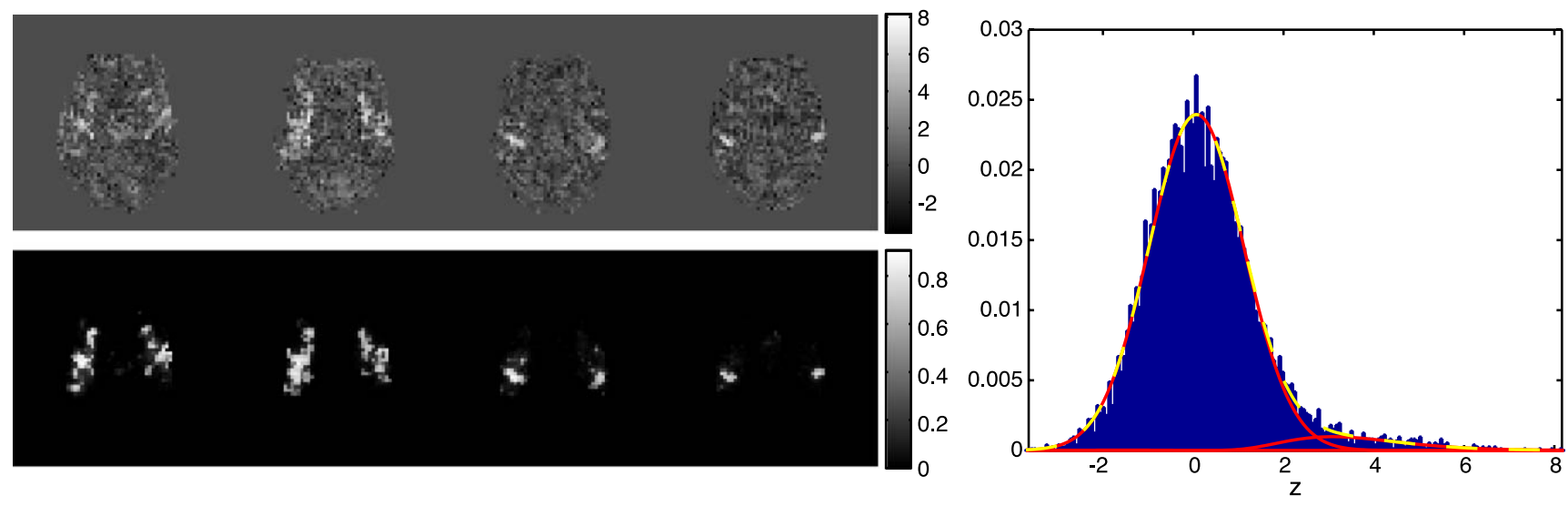

Fig. 11. Pain single-event condition. Results of applying the spatial mixture modelling on the unconstrained HRF model. (top) Pseudo-z-statistic spatial maps. (bottom) Probability of being in the activation class. (right) Mixture model fit to histogram of pseudo-z-statistics.

independent a posteriori. Therefore, we maintain a combined unfactorised posterior for the two parameters $\beta_{i}$ and $\bar{\beta}_{i}$. However, we have factorised the noise parameter posteriors from the regression parameter posteriors. This assumption helps to make inference tractable using Variational Bayes. Penny et al. (2003) discuss the implications of doing this and show that the error induced by this assumption is negligible for inferring on FMRI data. We also show later (in "Artificial null data") that there is negligible error induced when inferring on artificial data.

At this point, the inference is still not fully tractable to Variational Bayes as we cannot derive the update equations for $q\left(B_{i} \mid y\right)$. To overcome this we rewrite the prior in Eq. (22) as:

$\beta_{i e} \sim \operatorname{MVN}\left(\bar{\beta}_{i e} m, \phi_{\bar{\beta}_{i e}}^{-1} C^{-1}\right)$

where the utility parameter, $\phi_{\bar{\beta}_{i e}}$, is updated as a point estimate equal to 1 over the current expected value of $\bar{\beta}_{i e}^{2}$ :

$\phi_{\bar{\beta}_{i e}}=E\left[\bar{\beta}_{i e}^{-2}\right]^{-1}=R_{i e} \mu_{\bar{\beta}_{i e}}^{2}+\left(\Lambda_{B_{i}}^{-1}\right)_{\bar{\beta}_{i e}}$

where $R_{i e}$ is defined by the relationship $\bar{\beta}_{i e}=R_{i e} B_{i}$, and $\left(\Lambda_{B i}^{-1}\right) \bar{\beta}_{i e}$ is the current marginal covariance of $\bar{\beta}_{i e}$. The approximate posterior distributions are now tractable to Variational Bayes. The update rules for the approximate posterior distributions, which iteratively maximises the free energy in Eq. (18), are given in Appendix B.

We can perform standard inference questions on the marginal posterior over $\beta_{e}$, in the same way that we do for the standard use of basis functions in the GLM (i.e., using $f$-contrasts; see "fcontrasts" section). We test the accuracy of the posterior approximations presented in this section using null artificial data in the "Artificial null data" section.

The Variational Bayes inference requires approximately 10 iterations and takes approximately $15 \mathrm{~min}$ (for a whole brain: inplane resolution $4 \mathrm{~mm}$, slice thickness $7 \mathrm{~mm}$, and 180 volumes) on a $2 \mathrm{GHz}$ Intel PC.

\section{Initialisation}

We do not need to initialise the approximate distribution parameters of $q\left(\beta_{i}, \bar{\beta}_{i} \mid y\right)$. This is because we can initialise the other approximate distribution parameters and then update $q\left(\beta_{i}, \bar{\beta}_{i} \mid y\right)$ first. To allow us to provide sensible initialisation of the other approximate distributions, $q\left(\phi_{\varepsilon} \mid Y\right)$ and $q\left(a_{p}\right)$, we use the model with the autoregression parameters set to zero $\left(a_{p}=0\right)$ and with the HRF constraints removed $(m=0$ and $C=I)$. This means we can use the
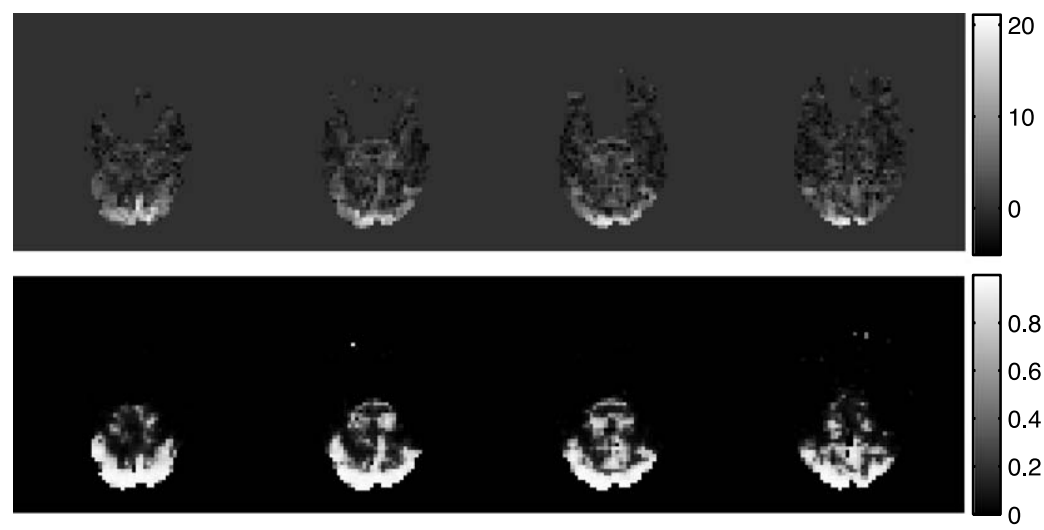

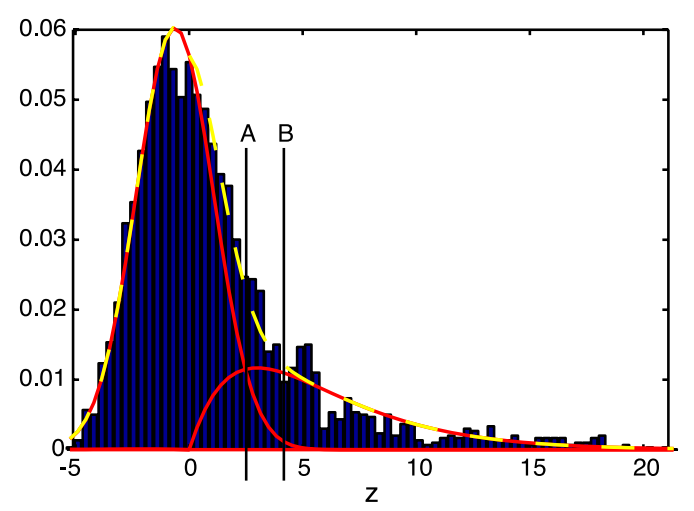

Fig. 12. Visual condition. Results of applying the spatial mixture modelling on the constrained HRF model. (top) Pseudo-z-statistic spatial maps. (bottom) Probability of being in the activation class. (right) Mixture model fit to histogram of pseudo-z-statistics ('A' and 'B' mark the pseudo-z-statistics for which voxels with greater pseudo-z-statistics have higher probability of being active than nonactive for the constrained HRF model and unconstrained HRF model, respectively). 



Fig. 13. Pain single-event condition. Results of applying the spatial mixture modelling on the constrained HRF model. (top) Pseudo-z-statistic spatial maps. (bottom) Probability of being in the activation class. (right) Mixture model fit to histogram of pseudo-z-statistics ('A' and 'B' mark the pseudo-z-statistics for which voxels with greater pseudo-z-statistics have higher probability of being active than nonactive for the constrained HRF model and unconstrained HRF model, respectively).

standard ordinary least squares (OLS) voxelwise frequentist solution to the GLM to get:

$\hat{\beta}_{i}=\left(x^{T} x\right)^{-1} x^{T} y_{i}$

$\hat{\eta}_{i t}=y_{i t}-x_{t} \hat{\beta}_{i}$

and

$S_{i}=\sum_{t}\left(\hat{\eta}_{i t}\right)^{2}$

The approximate distribution, $q\left(\phi_{\varepsilon_{j}} \mid Y\right)$, is set using frequentist results. The mean is set to $(T-K)=S$ and the variance to $2(T-$ $K)=S^{2}$. This mean and variance gives the approximate distribution parameters $b_{\varepsilon_{i}}$ and $c_{\varepsilon_{i}}$ (see Appendix A for the conversion).
We initialise $q\left(a_{p} \mid y\right)$ by using the frequentist solution to Eq. (2), where $\eta_{i}$ is set to the residuals, $\hat{\eta}_{i}$. This gives:

$\mu_{a i}=\left(\tilde{\eta}_{i} \tilde{\eta}_{i}^{T}\right)^{-1} \tilde{\eta}_{i} \hat{\eta}_{0}$

$\Lambda_{a i}=\sigma_{e}^{2}\left(\tilde{\eta} \tilde{\eta}^{T}\right)^{-1}$

where $\hat{\eta}_{i}=\left[\hat{\eta}_{i 1} \ldots \hat{\eta}_{i P}\right], \hat{\eta}_{i p}=\left[\hat{\eta}_{i(p+1)} \ldots \hat{\eta}_{i T}\right]^{T}$ and where:

$\sigma_{e_{i}}^{2}=\sum_{t}\left(\eta_{i t}-\eta_{i(t-p)} \mu_{a i}\right)^{2}$

We also initialise $b_{a_{p}}=1, c_{a_{p}}=1$ and $\phi \bar{\beta}_{k}=1$.
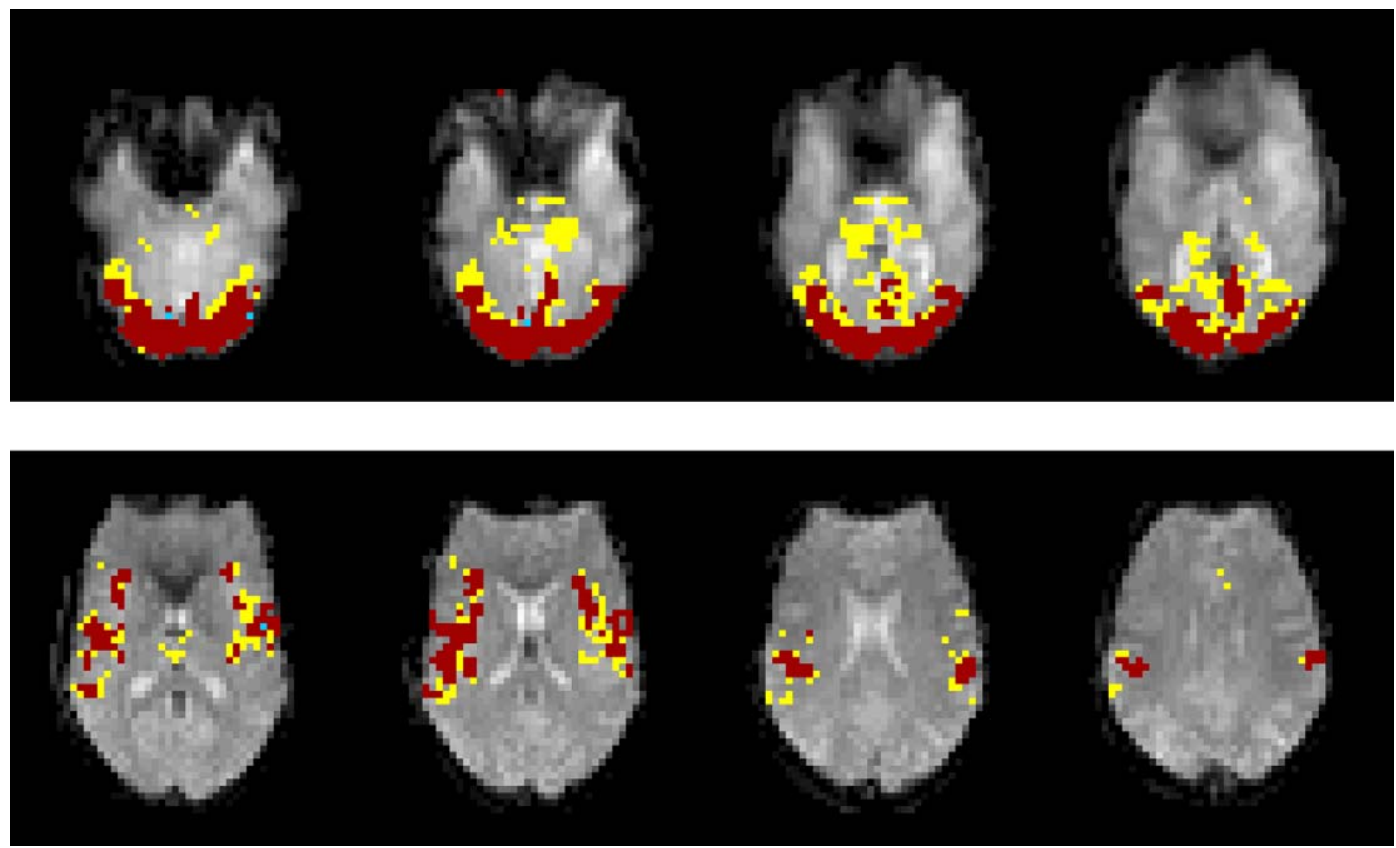

Fig. 14. Difference in voxel classification between the constrained HRF model and the unconstrained HRF model. (red) voxels are active for both models; (blue) voxels are active for just the unconstrained HRF model; and (yellow) voxels are active for just the constrained HRF model. Voxels are classified as activating with probability of being in the activation class greater then 0.5. (top) Visual boxcar dataset. (bottom) Pain single-event dataset. 


\section{$f$-contrasts}

Variational Bayes gives us an approximation to the posterior distribution, $p\left(\beta, a, \phi_{\varepsilon} \mid y\right)$. From this we can obtain the approximate marginal posterior distribution, $q(\beta, \bar{\beta} \mid Y)$, as being a multivariate Normal distribution (Eq. (25)). If we write this as:

$$
\left[\begin{array}{c}
\beta_{i} \\
\bar{\beta}_{i}
\end{array}\right] \mid y \sim \operatorname{MVN}\left(\left[\begin{array}{l}
\mu_{\beta_{i}} \\
\mu_{\bar{\beta}_{i}}
\end{array}\right],\left[\begin{array}{cc}
\Lambda_{\beta_{i}} & \Lambda_{\beta_{i} \bar{\beta}_{i}} \\
\Lambda_{\beta_{i} \bar{\beta}_{i}} & \Lambda_{\bar{\beta}_{i}}
\end{array}\right]\right)
$$

We can marginalise to get the marginal distribution over the regression parameters, $q\left(\beta_{i} \mid Y\right)$, as:

$\beta_{i} \mid y \sim \operatorname{MVN}\left(\mu_{\beta_{i}}, \Lambda_{\beta_{i}}\right)$

We can now use the marginal distribution in Eq. (33) to perform inference. In this paper we take the approach of using the $f$-contrast

\section{(a) Unconstrained}

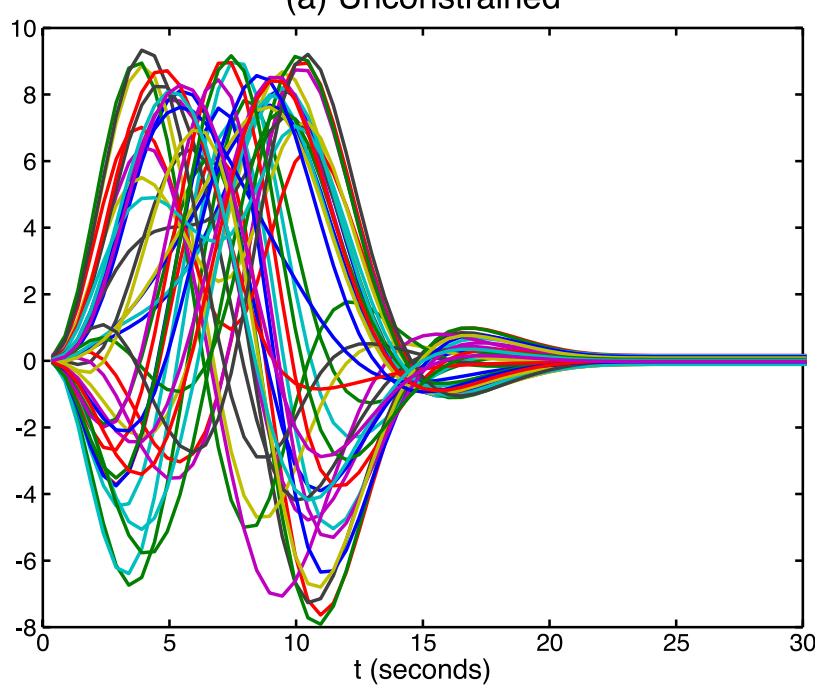

(b) Constrained

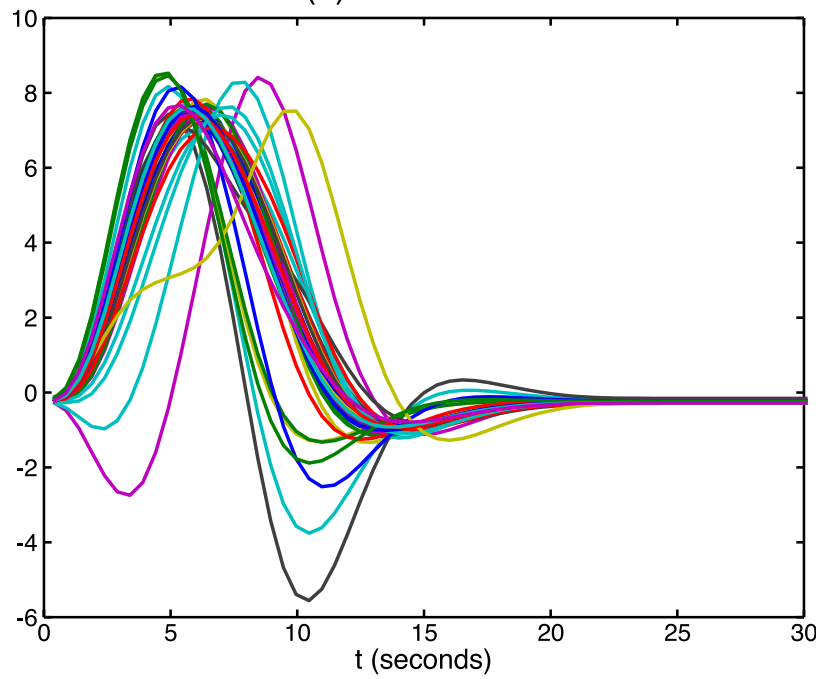

Fig. 15. Samples from the marginal posterior of the HRF at a single voxel, which is not activating in the pain experiment. (a) Unconstrained with $m=0$ and $C=I$ (b) Constrained with $m=\tilde{m}$ and $C=\tilde{C}$. (a) Unconstrained

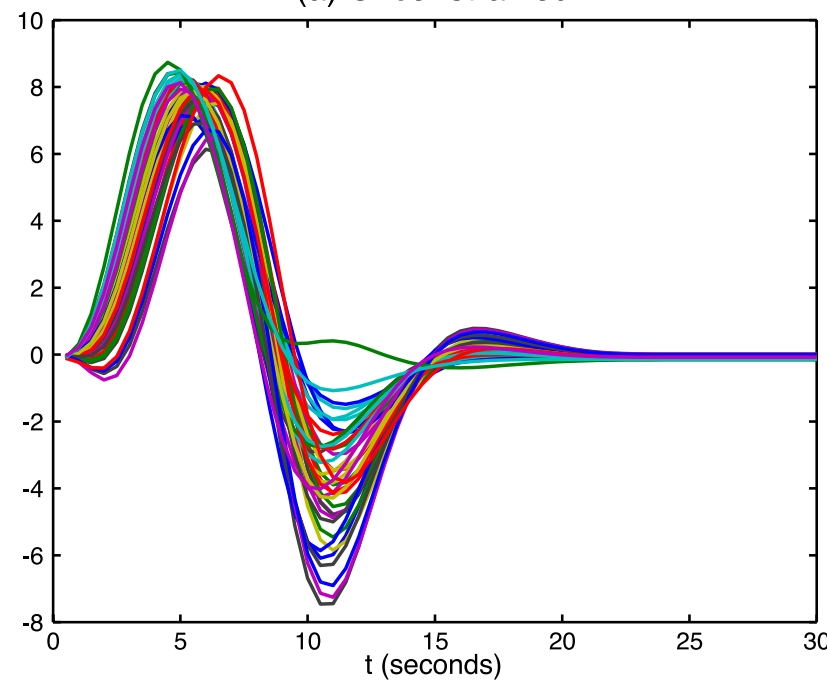

(b) Constrained



Fig. 16. Samples from the marginal posterior of the HRF at a single voxel, which is strongly activating in the pain experiment. (a) Unconstrained with $m=0$ and $C=I$ (b) Constrained with $m=\tilde{m}$ and $C=\tilde{C}$.

framework traditionally used with basis functions in the frequentist GLM framework (Josephs et al., 1997).

If $c$ is a $\left(N_{e} N_{b}\right) \times J$ vector representing an $f$-contrast, we can use the $f$-contrast framework to compute the normalised power explained by the $f$-contrast:

$f=\left(\mu_{\beta_{i}}^{T}\right) c\left(c^{T} \Lambda_{\beta_{i}} c\right)^{-1} c^{T} \mu_{\beta_{i}} / J$

with degrees of freedom $J$ and $\infty$. As with the use of basis functions in the frequentist GLM framework (Josephs et al., 1997), we lose directionality when doing an $f$ test. That is, we only investigate the power explained by linear combinations of the basis function parameters, regardless of the direction (i.e., whether it is an activation or deactivation). This means that we only look at the positive tail of the $f$-distribution to find both activations and deactivations.

The alternative to doing a test on $\beta_{i}$ would be to ask "What is the probability that $\bar{D}_{i e}$ is greater than zero?" Recall from Eq. (11) that $\bar{D}_{i e}$ represents the HRF size (activation height). Can we recover the 
activation height, $\bar{D}_{i e}$, from the parameters we infer upon, $\beta_{i e}$ and $\beta_{i e}^{-}$? To do this, we can rewrite Eqs. (20) and (21) to get:

$\bar{D}_{i e}=\bar{\beta}_{i e}\left(\sum_{b} \frac{\beta_{i e}^{2}}{N_{b} \bar{\beta}_{i e}^{2}}\right)^{1 / 2}=\operatorname{sign}\left(\bar{\beta}_{i e}\right)\left(\sum\left(\beta_{i e}^{2} / N_{b}\right)\right)^{1 / 2}$

Note that the term $\left(\sum\left(\beta_{i e}^{2} / N_{b}\right)\right)^{1 / 2}$ is the power we are testing when we do the $f$ test on $\beta_{i}$. Eq. (35) tells us that we can use the sign of $\beta_{i e}$ to give the sign of $\bar{D}_{i e}$, and therefore the direction of the activation.

We could look to derive the posterior probability of the normalised power explained by the $f$-contrast in Eq. (34). Instead, the approach we take in this paper is to convert them to pseudo-z-statistics and then perform spatial mixture modelling on the spatial map of pseudo- $z$-statistics as described later. The $f$-to- $z$ transform is carried out by doing an $f$-to- $p$-to- $z$ transform (i.e., by ensuring that the probabilities in the tails are equal under the $f$ - and $z$-distributions for the $f$ - and $z$ statistics).

We refer to them as pseudo-z-statistics as they are not necessarily Normally distributed with zero mean and standard deviation of one under the null hypothesis. This is because they have been obtained by performing Bayesian inference. Whether or not Bayesian inference produces the same null distribution as that in frequentist inference will depend on the form of prior used. As we shall see in "Artificial null data" and as we would expect (Penny et al., 2003), if we use noninformative priors we do get approximate equivalence between frequentist and Bayesian inference. However, when we use constrained HRF shape priors, we get a different distribution under the null hypothesis. We will see later how we can adjust to this different inference, and take advantage of the extra sensitivity it offers, by using spatial mixture modelling.

\section{Artificial null data}

Before we consider the use of spatial mixture modelling on the pseudo-z-statistics representing the normalised power explained by an $f$-contrast, we investigate the empirical null distributions produced from artificial data using the Variational Bayesian inference previously described.

\section{Methods}

We use artificial null data generated from the model, with $\beta=$ 0 and $a_{p}=0$. The stimulus is taken to be a square wave with period of $60 \mathrm{~s}$. We have $T=200$ scans, a TR of $3 \mathrm{~s}$ and use an HRF resolution of $\rho=6$. Using these values we generate time series for 10,000 voxels. We then fit the model with an autoregressive order of $P=4$ and use a basis set of the top 3 eigenHRFs shown in Fig. 3. We use two different models, one with no HRF constraints ( $m=0$ and $C=I)$ and one with HRF constraints $[m=\tilde{m}$ and $C=\tilde{C}$ as given in Eq. (17)]. We use an $f$-contrast of $[1,0,0 ; 0,1,0 ; 0,0,1]$.

\section{Results}

Fig. 5(a) shows the histogram of pseudo-z-statistics obtained for the two different models with and without HRF constraints using the Variational Bayesian inference. Fig. 5(b) shows the $\log$ probability-log probability plots. These show plots of (nominal/ theoretical) frequentist FPR against that obtained empirically.

The nominal/theoretical FPR is only applicable to the unconstrained HRF model, as we then have noninformative priors and we would expect the Bayesian inference to be equivalent to frequentist inference. Accordingly, the log probability-log probability plot shows good correspondence between the empirically obtained probabilities under the tail for a given $z$-statistic, and that which we expect from frequentist theory, for the unconstrained HRF model.

Recall that to make the inference tractable under Variational Bayes, we introduced a utility parameter, $\phi_{\beta_{i e}}$, which we update with point estimates an approximation that may have effected the marginal posterior over $\beta_{i e}$. The fact that we obtain good correspondence here between our inference and the (for this model, known to be correct) frequentist results provides some validation that the marginal posterior over $\beta_{e}$ is not significantly affected.

Unlike the unconstrained model, we would not expect the constrained HRF model to give pseudo-z-statistics that conform to frequentist theory. This is because we now have informative HRF shape priors causing Bayesian inference to be different to frequentist inference. The log probability-log probability plot shows that we get probabilities under the tail for a given $z$-statistic much smaller empirically than if the frequentist GLM solution held true. The histogram in Fig. 5(a) shows that this is due to a large shift in the histogram to lower pseudo-z-statistics (the mode is at about $z=-1$ ). The HRF constraints reduce the power in those voxels where the linear combinations of the basis functions do not give sensible HRF shapes; this produces a shift in the nulldistribution histogram. When we have activating voxels with HRF shapes, which are not penalised by the HRF prior constraints, then the pseudo-z-statistics will not be reduced. We therefore have extra sensitivity when we use Bayesian inference with informative priors constraining the HRF shape.

\section{Spatial mixture modelling}

Using Variational Bayesian inference, we have produced a spatial map of pseudo-z-statistics. Since the pseudo-z-statistics for the constrained HRF model give smaller empirical null distribution probabilities than the nominal FPR, we could use the pseudo-z-statistics and infer on them using frequentist probabilities without violating our nominal FPR. However, we would not be taking full advantage of the extra sensitivity of the Bayesian inference when we have informative priors constraining the HRF shape.

Therefore, we look to infer on the spatial map of pseudo-zstatistics using the technique of spatial mixture modelling. In mixture modelling, we model the spatial map as being made up of voxels that are classified as either activating or nonactivating (Everitt and Bullmore, 1999). The activation and deactivation classes have different distributions, which describe probabilistically the pseudo-z-statistic values we expect in that class. Since we are estimating the nonactivating distribution from the data, we can adjust to the shifted nonactivation distribution demonstrated in Fig. 5.

In spatial mixture modelling, we augment this histogram information with spatial regularisation of the classification (Hartvig and Jensen, 2000; Woolrich et al., submitted for publication). Note 
that this has nothing to do with the way in which we spatially regularise the temporal autocorrelation coefficients in the "Autoregressive parameters spatial prior" section. Instead, we spatially regularise the classification of activating or nonactivating voxels. This has been implemented in Woolrich et al. (submitted for publication), within a completely adaptive fully Bayesian framework. Importantly, this approach adaptively determines the amount the classification is spatially regularised.

Fig. 6 shows the null distribution of pseudo-z-statistics resulting from the constrained HRF analysis on the artificial null data in the previous section. This distribution is not Normal; in particular it is asymmetric. We therefore model the nonactivation distribution in the mixture modelling as a flipped and shifted Gamma distribution. Fig. 6 shows the fit of a flipped and shifted Gamma distribution to the null distribution histogram. Recall that with an $f$-contrast we only look at the positive tail of the distribution to find both activations and deactivations. Therefore, we model the activations and deactivations as a single class in the spatial mixture modelling using a straightforward Gamma distribution.

\section{FMRI data}

\section{Methods}

We use two different datasets. The first is a boxcar visual experiment with a reversing checkerboard boxcar stimulus (30-s on, 30-s off). The second is a single-event pain experiment, for which the stimulus was a thermal noxious stimuli of $3 \mathrm{~s}$ duration administered to the dorsum of the volunteer's left hand using an electrical resistor to generate heat with varying ISI (between 30 and $50 \mathrm{~s})$.

For both experiments, echo planar images (EPI) were acquired using a 3-T system with TR $=3 \mathrm{~s}$, time to echo (TE) $=30 \mathrm{~ms}$, inplane resolution $4 \mathrm{~mm}$ and slice thickness $7 \mathrm{~mm}$. The first four scans were removed and the data was motion corrected using MCFLIRT (Jenkinson et al., 2002) and high-pass filtered as described in Woolrich et al. (2001). The data is not spatially smoothed.

We use an HRF resolution of $\rho=6$ (i.e., $0.5 \mathrm{~s}$ ). We then fit the model with an autoregressive order of $P=4$ and use a basis set of the top 3 eigen-HRFs shown in Fig. 3. We use two different models, one with no HRF constraints $(m=0$ and $C=I)$ and one with HRF constraints $[m=\tilde{m}$ and $C=\tilde{C}$ as given in Eq. (17)]. We use an $f$-contrast to pick out the linear combinations of the basis functions. The resulting $f$-statistics are $f$-to- $z$ converted to produce spatial maps of pseudo-z-statistics. The fully adaptive mixture modelling described in the previous section is then used to provide probabilities of a voxel being activated.

\section{Results}

Fig. 7 shows the posterior means of the AR parameters from the single-event pain dataset for two different models: with nonspatial and with spatial MRF AR parameter priors. For the model with spatial MRF priors, the Variational Bayesian inference adaptively determines the amount of spatial regularisation to impose for each of the autoregressive parameter MRFs separately. The spatial maps for $p=1$ are very similar, but as we increase $p$ the MRF spatial regularisation increases. This shows how the adaptive determination of the amount of spatial regularisation automatically adjusts to avoid overfitting of the high-order autoregressive parameters. In terms of inference on the normalised power of the $f$-contrasts, Fig. 8(a) suggests that avoiding overfitting and spatially regularising the AR parameters does make a significant difference to the pseudo-zstatistics.

Fig. 9 shows the histogram of pseudo-z-statistics obtained for the two different models with and without HRF constraints. For both conditions, we can see how the right hand tail, i.e., those voxels that are strongly activating, is relatively unaffected. Whereas, the main body of the histogram, i.e., the background nonactivating voxels, is shifted to the left in the same way that it was for the null artificial data.

Figs. 10 and 11 show the results of applying the spatial mixture modelling on the unconstrained HRF model. Figs. 12 and 13 show the results for the constrained HRF model. Fig. 14 shows the difference in voxel classification between the constrained HRF model and the unconstrained HRF model. This difference highlights the increased sensitivity. With the constrained HRF model, smaller strength activating voxels have increased probability of being in the activation class. This is because the nonactivating class distribution is shifted to lower pseudo-z-statistics when we use the constrained HRF model.

Figs. 15 and 16 show samples from the marginal posterior of the HRF at a voxel that is not activating and a voxel that is strongly activating, respectively, in the pain experiment. In particular, Fig. 15 highlights the difference between the unconstrained and constrained HRF models for a nonactivating voxel. Whereas, in Fig. 16 , the HRF shape conforms to prior expectations; hence, there is little difference between the unconstrained and constrained HRF models for a strongly activating voxel.

\section{Conclusions}

We have proposed a technique for firstly choosing a basis set, and then the means to constrain the subspace spanned by the basis set to only include sensible HRF shapes within a GLM framework. We can carry out inference using Variational Bayes, which also performs adaptive spatial regularisation of temporal autocorrelation. Constraining the subspace spanned by the basis set allows for far superior separation of activating voxels from nonactivating voxels in FMRI data. We used spatial mixture modelling to produce final probabilities of activation, and demonstrated on FMRI data the increased sensitivity produced.

\section{Discussion}

In a previous work Friman et al. (2003) also looked to constrain the possible linear combinations of the basis set, but within the canonical correlation analysis (CCA) framework. However, they only looked to constrain the linear combination coefficients to be positive. In this work we apply a more complete constraint by fitting a multivariate Normal distribution to describe the desired constrained space probabilistically. A big limitation of the work in Friman et al. (2003) is that they did not address the issue of how to threshold the resulting correlations of the CCA. In contrast, the framework in this paper is the Variational Bayesian GLM framework first used in FMRI by Penny et al. (2003). This framework has the advantage that it takes into account important issues such as temporal autocorrelation in FMRI and at the same time 
intrinsically produces approximate probability distributions from which inference can take place.

The HRF modelling in this paper all assumes linearity of the HRF. Friston et al. (1998b) produced compelling work addressing the use of basis functions for nonlinear HRF modelling using Volterra series. They model the first- and second-order kernels using Gamma basis functions in a frequentist inferred GLM. In Friston et al. (2000), they derive Volterra kernels from the balloon model (Buxton et al., 1998) and fit them to empirically found Volterra kernels from the frequentist inferred GLM of Friston et al. (1998b). In Friston (2002), they infer on the balloon model parameters directly from the FMRI data in a Bayesian framework. Within the Bayesian framework they can incorporate priors on the balloon model parameters deduced empirically in Friston et al. (2000). This incorporation of the prior information from previous empirical evidence will constrain the balloon model parameters in the same way we constrain the HRF shape basis function parameters in this work. One area of future work is to extend the Variational Bayesian inference in this paper to deal with second-order Volterra kernel basis functions and nonlinearities in ways related to the work of Friston et al. (1998b).

\section{Acknowledgments}

The authors would like to acknowledge support from the UK MRC and EPSRC. Thanks must also go to Heidi Johansen-Berg, Mark Jenkinson and Christian Beckmann for their valuable input. Thanks to Richard Wise, Richard Rogers and Debbie Painter of the Nuffield Department of Anaesthetics, John Radcliffe Hospital, Oxford University for the pain dataset.

\section{Appendix A. Gamma distribution}

$x$ has a two-parameter gamma distribution, denoted by $G a(b, c)$, with parameters $b$ and $c$, if its density is given by:

$f_{G a}(x ; b, c)=\frac{1}{\Gamma(c)} \frac{x^{c-1}}{b^{c} e^{-x / b}}$

where $\Gamma(c)$ is the Gamma function. Note that a gamma distribution has mean $=b c$ and variance $=b^{2} c$.

\section{Appendix B. Variational Bayes updates}

We define

$B_{i}=\left[\begin{array}{c}\beta_{i} \\ \hat{\beta}_{i}\end{array}\right]$

and

$\beta_{i}=Q B_{i}$

$\beta_{i e}=Q_{i e} B_{i}$

$\bar{\beta}_{i e}=R_{i e} B_{i}$

\section{B.1. Regression coefficient updates}

Here we give the update equation for the parameters of the regression coefficient distribution $q\left(\beta_{i} ; \bar{\beta}_{i} \mid y\right)=\operatorname{MVN}\left(\mu_{B_{i}}, \Lambda_{B_{i}}\right)$ :

$\mu_{B_{i}}=F_{B_{i}}^{-1} E_{B_{i}}$

$\Lambda_{B_{i}}=F_{B_{i}}^{-1}$

where

$$
\begin{aligned}
F_{B_{i}}= & \gamma_{e_{i}} \sum_{t}\left\{Q^{T} x_{i}^{T} x_{t} Q+\sum_{p} \mu_{a_{p i}}\left(Q^{T} x_{t-p}^{T} x_{t-p} Q-2 Q^{T} x_{t}^{T} x_{t-p} Q\right)\right\} \\
& +\sum_{e}\left\{\phi_{\bar{\beta}_{e}}^{(i)}\left(Q_{e}^{T} C Q_{e}+R_{e}^{T} m^{T} C m R_{e}-2 Q_{e}^{T} C m R_{e}\right)\right\} \\
E_{B_{i}}= & \gamma_{e_{i}} \sum_{t}\left\{Q^{T} x_{t}^{T}\left(y_{i t}-\sum_{p} y_{i(t-p)} \mu_{a_{p i}}\right)\right. \\
& \left.+\sum_{p} \mu_{a_{p i}} Q^{T} x_{t-p}^{T}\left(y_{i t}-\sum_{p} y_{i(t-p)} \mu_{a_{p i}}\right)\right\} \\
\gamma_{e_{i}}= & \frac{\Gamma\left(b_{e_{i}}\right) \Gamma\left(c_{e_{i}}+1\right)}{\Gamma\left(c_{e_{i}}\right)}
\end{aligned}
$$

\section{B.2. Autoregressive parameter updates}

Here we give the update equation for the parameters of the autoregressive parameter distribution $q\left(a_{p} \mid y\right)=N\left(\mu_{a_{p}}, \Lambda_{a_{p}}\right)$ :

$\mu_{a_{p}}=F_{a_{p}}^{-1} E_{a_{p}}$

$\Lambda_{a_{p}}=F_{a_{p}}^{-1}$

where

$F_{a_{p}}=\sum_{t}\left(w_{t p}^{T} S_{e} w_{t p}\right)+\gamma_{a_{p}} D$

$E_{a_{p}}=\sum_{t} w_{t p}^{T} S_{e}\left(y_{t}-\sum_{q \neq p} w_{t q} \mu_{a_{q}}-x_{t} Q \mu_{B}\right)$

where $S_{e}=\operatorname{Diag}\left(\gamma_{\varepsilon_{i}}\right)$ and $w_{t_{p}}=y_{t-p}-x_{t-p} Q_{\mu_{B}}$

\section{B.3. MRF precision updates}

Here we give the update equation for the MRF precision parameter distribution $q\left(\phi_{a_{p}} \mid y\right)=G a\left(b_{\phi a_{p}}, c_{\phi a_{p}}\right)$ :

$\frac{1}{b_{a_{p}}}=\frac{1}{2}\left(m_{a_{p}}^{T} D m_{a_{p}}+\operatorname{Trace}\left(\Lambda_{a_{p}} D\right)\right)+\frac{1}{b_{a_{0}}}$

$C_{a_{p}}=N / 2+c_{a_{0}}$

we define $\gamma_{a_{p}}$ as:

$\gamma_{a_{p}}=\frac{\Gamma\left(b_{a_{p}}\right) \Gamma\left(c_{a_{p}}+1\right)}{\Gamma\left(c_{a_{p}}\right)}$

Even though the matrix $D$ is very sparse, the computation of the Trace $\left(\Lambda_{a_{p}} D\right)$ term can be very expensive to compute. In particular, this is because $\Lambda_{a_{p}}=F_{a_{p}}^{-1}$ and $F_{a_{p}}$ is a $N \times N$ matrix whose inverse would be very computationally expensive to 
compute. Therefore, instead of computing this inverse, we can compute $x$ in the linear equation:

$$
F_{a_{p}} x=D
$$

Since $D$ is a positive symmetric definite matrix, we can take advantage of the conjugate gradient techniques described in Golub and Van Loan (1996). At each iteration of the conjugate gradient search for $x$, we only need to perform one matrix multiplication of $F_{a_{p}} x$. The conjugate gradient approach is far quicker than solving for the inverse of $F_{a_{p}}$ and then multiplying by $D$.

The conjugate gradient technique takes in an initial guess of $x$. Hence, as we iterate through the Variational Bayes updates of our approximate posterior distributions, we can store the value of $x$ from the previous conjugate gradient solution from the previous update of $q\left(\phi_{a_{p}} \mid y\right)$, and use it as the initialisation of the conjugate gradient search for $x$ at the next update of $q\left(\phi_{a_{p}} \mid y\right)$. After the first Variational Bayes iteration, this makes subsequent conjugate gradient searches very quick to converge.

\section{B.4. Noise precision updates}

Here we give the update equation for the parameters of the noise precision distribution $q\left(\phi_{e_{i}} \mid y\right)=G a\left(b_{e_{i}}, c_{e_{i}}\right)$ :

$$
\begin{aligned}
\frac{1}{b_{e_{i}}}= & \frac{1}{2}\left(\sum_{t}\left(y_{i t}-x_{t} Q \mu_{B_{i}}-\sum_{p}\left(y_{i(t-p)}-x_{t-p} Q \mu_{B_{i}}\right) a_{p i}\right)^{2}\right. \\
& +\operatorname{Trace}\left(\Lambda_{\beta_{i}}^{-1} \sum_{t} x_{t}^{T} x_{t}\right)+\sum_{p}\left\{( \Lambda _ { a _ { p } } ^ { - 1 } ) _ { i } \sum _ { t } \left(y_{i(t-p)}\right.\right. \\
& \left.\left.\left.-x_{t-p} Q \mu_{B_{i}}\right)^{2}\right\}\right)+\frac{1}{b_{e_{0}}}
\end{aligned}
$$

$c_{e_{i}}=(T-1) / 2+c_{e_{0}}$

we define $\gamma_{e_{i}}$ as:

$\gamma_{e_{i}}=\frac{\Gamma\left(b_{e_{i}}\right) \Gamma\left(c_{e_{i}}+1\right)}{\Gamma\left(c_{e_{i}}\right)}$

\section{References}

Boynton, G., Engel, S., Glover, G., Heeger, D., 1996. Linear systems analysis of functional magnetic resonance imaging in human V1. J. Neurosci. 16, 4207-4221.

Buxton, R., Wong, E., Frank, L., 1998. Dynamics of blood flow and oxygenation changes during brain activation: the balloon model. Magn. Reson. Med. 39, 855-864.

Cohen, M., 1997. Parametric analysis of fMRI data using linear systems methods. NeuroImage 6, 93-103.

Cressie, N., 1993. Statistics for Spatial Data. Wiley, New York.
Dale, A., Buckner, R., 1997. Selective averaging of rapidly presented individual trials using fMRI. Hum. Brain Mapp. 5, 329-340.

Everitt, B., Bullmore, E., 1999. Mixture model mapping of brain activation in functional magnetic resonance images. Hum. Brain Mapp. 7, $1-14$.

Friman, O., Borga, M., Lundberg, P., Knutsson, H., 2003. Adaptive analysis of fMRI data. NeuroImage 19 (3), 837-845.

Friston, K.J., 2002. Bayesian estimation of dynamical systems: an application to fMRI. NeuroImage 16,513-530.

Friston, K., Worsley, K., Frackowiak, R., Mazziotta, J., Evans, A., 1994. Assessing the significance of focal activations using their spatial extent. Hum. Brain Mapp. 1, 214-220.

Friston, K.J., Frith, C.D., Turner, R., Frackowiak, R.S.J., 1995. Characterizing evoked hemodynamics with fMRI. NeuroImage 2, 157-165.

Friston, K., Fletcher, P., Josephs, O., Holmes, A., Rugg, M., Turner, R., 1998a. Event-related fMRI: characterizing differential responses. NeuroImage 7, 30-40.

Friston, K., Josephs, O., Rees, G., Turner, R., 1998b. Nonlinear eventrelated responses in fMRI. Magn. Reson. Med. 39, 41-52.

Friston, K., Mechelli, A., Turner, R., Price, C., 2000. Nonlinear responses in fMRI: the balloon model, Volterra kernels, and other hemodynamics. NeuroImage 12, 466-477.

Genovese, C.R., 2000. A Bayesian time-course model for functional magnetic resonance imaging data (with discission). J. Am. Stat. Assoc. 95, $691-703$

Golub, G.H., Van Loan, C.F., 1996. Matrix Computations, third ed. Johns Hopkins Univ. Press, Baltimore.

Gössl, C., Auer, D., Fahrmeir, L., 2001. Bayesian modeling of the haemodynamic response function in BOLD fMRI. NeuroImage 14 (1), $140-148$.

Hartvig, N., Jensen, J., 2000. Spatial mixture modelling of fMRI data. Hum. Brain Mapp. 11 (4), 233-248.

Hossein-Zadeh, G., Ardekani, B., 2002. A signal subspace for modeling the hamodynamic response function in fMRI. Eighth Int. Conf. on Functional Mapping of the Human Brain. NeuroImage.

Jenkinson, M., Bannister, P., Brady, J., Smith, S., 2002. Improved optimisation for the robust and accurate linear registration and motion correction of brain images. Neurolmage 17 (2), 825-841.

Jordan, M., 1999. Learning in Graphical Models. MIT Press.

Josephs, O., Turner, R., Friston, K., 1997. Event-related fMRI. Hum. Brain Mapp. 5, 1-7.

Penny, W.D., Kiebel, S., Friston, K.J., 2003. Variational Bayesian inference for fMRI time series. Neurolmage 19 (3), 1477-1491.

Woolrich, M., Ripley, B., Brady, J., Smith, S., 2001. Temporal autocorrelation in univariate linear modelling of FMRI data. NeuroImage 14 (6), $1370-1386$.

Woolrich, M.W., Behrens, T.E.J., Beckmann, C.F., Smith, S.M., 2004a. Mixture models with adaptive spatial regularisation for segmentation with an application to FMRI data. Oxford Centre for Functional Magnetic Resonance Imaging of the Brain, Department of Clinical Neurology. Technical Report TR04MW1. Oxford University, Oxford, UK (Available at www.fmrib.ox.ac.uk/analysis/techrep for downloading, submitted for publication).

Woolrich, M., Jenkinson, M., Brady, J.M., Smith, S.M., 2004b. Fully Bayesian spatio-temporal modelling of FMRI data. IEEE Trans. Med. Imaging 22 (2) (in press).

Worsley, K., Liao, C., Aston, J., Petre, V., Duncan, G., Morales, F., Evans, A., 2002. A general statistical analysis for fMRI data. NeuroImage 15 (1), $1-15$. 